\section{A software system for recording and analyzing transient evoked potential data with an Apple IIe computer}

\section{JOHN A. BARO and STEPHEN LEHMKUHLE}

School of Optometry, University of Missouri-St. Louis St. Louis, Missouri

The software package described here was designed to digitize transient evoked potential data from two channels simultaneously and to analyze the data using an Apple Ile computer and a relatively inexpensive hardware configuration. Individual modules provide several data collection paradigms, a number of data analyses, and display capabilities. The system is capable of accommodating a variety of hardware configurations. The data collection module independently controls the presentation of two stimuli and averages, displays, and records the data. The summary/plotting module allows for simple analyses of the data (e.g., peak-trough amplitudes and latencies) and provides the ability to draw waveforms on a pen plotter. The analysis module performs a model referenced identification (MRI) analysis on individual waveforms (see O'Connor, Tasman, Simon, \& Hale, 1983). This involves fitting waveforms with the most appropriate damped sinewave component(s). The result is a quantification of the raw data, in which each component is completely described by four parameters.

All programs are completely menu driven and fully automated; familiarity with experimental rationale or procedures is not necessary to operate this system. Utility programs are provided so that routine disk and file operations may be performed without exiting the data collection/analysis system. Programs are written in assembly language and compiled Applesoft BASIC (compilation of BASIC programs is necessary to utilize all available memory and to increase the speed of execution). BASIC programs were written under and require ProDOS 8, and machine language routines were written under DOS 3.3 and then translated into ProDOS.

\section{Program Description and Specifications}

Data Collection Module. Data collection software provides an environment for acquiring data under a variety of stimulus conditions and experimental paradigms. Electrodes placed on the scalp (or on the cornea, or implanted in the brain of animal subjects) to record neural activity evoked by transient patterns are sampled at up to $12 \mathrm{KHz}$ (depending on hardware configuration), and the recorded waveform is displayed on the computer's video monitor. ${ }^{1}$ Two stimulus patterns can be controlled independently by varying the output voltage of two digital-to-analog chan-

Address correspondence to John A. Baro, School of Optometry, University of Missouri-St. Louis, St. Louis, MO 63121. nels, and stimuli can be turned on and off at a wide range of times before, during, and after sampling. This permits a variety of stimulus timing combinations. All subject information and stimulus/recording parameters are initially set when the program begins and can be changed at any time.

During each sweep, 256 8-bit values are sampled; successive sweeps are added together, and an averaged waveform is displayed at user-specified intervals. The user also specifies an artifact-rejection value so that unwanted, artifactual large-amplitude signals can be excluded from the averaged data. Sampling continues only when a key (or a button on a joystick) is depressed to avoid collecting data when the subject is not attending to the display. Sampling can also be terminated and restarted at any time. Following a predetermined number of sweeps, the raw data can be saved to disk.

Summary/Plotting Module. Summary software allows a number of manipulations to be performed on the raw data. After the user selects one or more files, the program displays them individually or superimposed for comparison between waveforms. Options include scaling of the waveform or zooming in on a portion of the waveform by manipulating an on-screen cursor. Summary information provides measures of peak-to-trough amplitude and latencies of both positive and negative deflections relative to stimulus onset/offset within a defined window. A hardcopy of the waveform can also be obtained from an $x-y$ pen plotter. When the user is finished with a waveform, the next selected file is loaded and displayed.

MRI Analysis Module. This module performs an MRI analysis (see O'Connor et al., 1983) on selected waveforms. This analysis permits a description of a given waveform in terms of up to seven damped sine-wave components that, when summed, approximate the original waveform. Each component is completely described by four parameters and is defined by

$$
y(t)=A e^{-\beta \tau} \sin (\omega \tau) ; \tau=t-t_{s},
$$

where $A$ is the amplitude, $\omega$ is the frequency, $\beta$ is the inverse of the time constant of the exponentially decaying envelope, and $t_{z}$ is the latency between the stimulus and the beginning of the response. The analysis involves decomposing each waveform into its components and fitting each component individually. With the original waveform on screen, the user can approximate frequency, latency, and the time constant by moving a cursor to specified portions of the waveform (e.g., frequency is estimated by positioning the cursor at the first peak). The best fitting component is then found by varying each of the four variables until the smallest residual (i.e., the difference between the original and the obtained component) is obtained. The user may specify the range and number of values used for each of the variables. After the first component is obtained, the user may attempt to 
fit a second component to the resulting residual and continue to fit additional components until the remaining residual is satisfactorily small. The individual components can then be summed and compared with the original waveform. An "auto run" option is also provided, so that a number of waveforms can be analyzed without user intervention.

\section{System Requirements}

The software system requires an Apple Ile computer with $128 \mathrm{~K}$ of RAM memory and an Image Writer II printer; a hard disk is strongly recommended. Additional hardware requirements are flexible; a number of possible configurations can easily be accommodated (utility software is provided for configuring the system). An 8-bit, memory-mapped digital-to-analog/analog-to-digital converter is needed and may reside in any slot (the A/D+D/A Card by Applied Engineering, Inc., P.O. Box 798, Carrollton, TX 75006, is recommended). To realize its full capability of stimulus control, an image generator with external control of two stimulus patterns is recommended (e.g., the Picasso, by Innisfree, Inc., P.O. Box 160, Cambridge, MA 02236). Other commonly used equipment necessary to complete the system includes a video display monitor, physiological amplifiers, and an $x-y$ plotter.

\section{Availability}

Compiled software and a complete reference manual are available from the authors for $\$ 12$ (please specify 3.5or 5.25-in. disk format). Documented source code is available upon request and requires the use of the Beagle Compiler by Beagle Bros Micro Software, Inc. (1990 Old Town Ave., San Diego, CA 92110) and an assembler (source code for the BASIC programs will not run under the Applesoft interpreter).

\section{REFERENCE}

O'Connor, S. J., Tasman, A., Simon, R. H., Hale, M. S. (1983). A model referenced method for the identification of evoked potential component wave forms. Electroencephalography \& Clinical Neurophysiology, 55, 233-237.

\section{NOTE}

1. The user should refer to the documentation accompanying the analog-to-digital board to determine the maximum sampling rate. Our hardware configuration permits sampling up to about $10 \mathrm{kHz}$. 